\title{
Simple recipes for compact remnant masses and natal kicks
}

\author{
Ilya Mandel ${ }^{1,2,3,4}$ and Bernhard Müller ${ }^{1,2}$ \\ 1 School of Physics and Astronomy, Monash University, Clayton, Vic. 3800, Australia \\ 2 The ARC Centre of Excellence for Gravitational Wave Discovery - OzGrav \\ 3 The ARC Centre of Excellence for All Sky Astrophysics in 3 Dimensions - ASTRO 3D \\ 4 Birmingham Institute for Gravitational Wave Astronomy and School of Physics and Astronomy, \\ University of Birmingham, B15 2TT, Birmingham, UK
}

Accepted 2020 September 28. Received 2020 September 25; in original form 2020 June 15

\begin{abstract}
Based on recent results from three-dimensional supernova simulations and semianalytical parametrised models, we develop analytical prescriptions for the dependence of the mass of neutron stars and black holes and the natal kicks, if any, on the presupernova carbon-oxygen core and helium shell masses. Our recipes are probabilistic rather than deterministic in order to account for the intrinsic stochasticity of stellar evolution and supernovae. We anticipate that these recipes will be particularly useful for rapid population synthesis, and we illustrate their application to distributions of remnant masses and kicks for a population of single stars.
\end{abstract}

Key words: supernovae: general - stars: neutron - stars: black holes

\section{INTRODUCTION}

Stars with initial masses above $\gtrsim 8 M_{\odot}$ end their lives as compact objects: neutron stars or black holes. The amount of mass ejected during the supernova explosion, which may accompany the collapse of the stellar core, determines the remnant mass. Meanwhile, an asymmetry in the explosion leads to a natal kick of the remnant. If the massive star was a component of a stellar binary, as most massive stars are (Sana et al. 2012), the mass loss and natal kick determine the future evolution of the system. Will it be disrupted by the explosion? Will it become an X-ray binary? Could it merge as a gravitational-wave source? Even for single stars, natal kicks play critical roles; for example, they determine the fraction of compact objects retained in globular clusters. Rapid stellar and binary population synthesis codes rely on recipes for predicting remnant masses and kicks from presupernova properties.

Detailed modelling of core-collapse supernovae of evolved massive stars is a long-standing problem in computational astrophysics (see, e.g. Janka 2012; Burrows 2013; Müller 2020, for reviews). Below, we touch on some of the challenges associated with ab-initio modelling.

On the other hand, there are no direct observations that could tie a pre-supernova stellar mass to the remnant mass or kick for an individual star, although a few supernova remnants such as Cas A allow for tentative remnant mass estimates with some information on the ejecta mass coming from light echoes (e.g., Hwang \& Laming 2012), and a few tens of supernova progenitors have been directly observed (Smartt 2015; Van Dyk 2017).
Consequently, remnant mass and kick predictions are typically based on either simple analytical models, extrapolations of numerical simulations, or on observational constraints on entire populations of stars. Hurley et al. (2000); Fryer et al. (2012) provide some the commonly used prescriptions for remnant masses, with more recent models available from, e.g., Müller et al. (2016) and Patton \& Sukhbold (2020). Remnant kick prescriptions for neutron stars are typically based on observed velocities of single pulsars (Lyne \& Lorimer 1994; Hansen \& Phinney 1997; Cordes \& Chernoff 1998; Arzoumanian et al. 2002; Hobbs et al. 2005; FaucherGiguère \& Kaspi 2006; Verbunt et al. 2017). However, using these prescriptions directly entails applying the same kick distributions to all neutron stars, regardless of pre-supernova properties, perhaps with scaled kicks for black holes (e.g., Fryer et al. 2012; Belczynski et al. 2012). Some population synthesis codes develop their own prescriptions, e.g., Belczynski et al. (2008) or Spera et al. (2015) for remnant masses and Vigna-Gómez et al. (2018) or Bray \& Eldridge (2016, 2018); Giacobbo \& Mapelli (2020) for natal kicks.

Here, we use recent findings from three-dimensional (3D) supernova simulations and parametrised onedimensional (1D) and one-zone models as a starting point to predict the remnant mass from the progenitor core mass. Unlike other models (see Clausen et al. 2015 for a notable exception), we do not attempt to deterministically prescribe a unique remnant mass, but rather account for the stochasticity in the pre-supernova stellar evolution and the stellar collapse itself by providing a probabilistic recipe. We use the same models to predict natal kicks, which are thus coupled to the explosion properties and the remnant 
masses. Our recipes have several tuneable parameters that are only approximately predicted by the numerical models. We propose that these should be calibrated by observations. We discuss the insights gained from supernova models in Section 2; introduce the parametrised recipes directly usable in population synthesis in Section 3; illustrate the consequences of these recipes for single stellar evolution in Section 4; and briefly summarise in Section 5 .

\section{PHYSICAL MODEL}

\subsection{Phenomenology of $1 \mathrm{D}$ and $3 \mathrm{D}$ supernova simulations}

In recent years, parametrised models and first-principle 3D simulations have made significant progress in establishing the relationship between the supernova progenitor, the explosion, and the properties of the remnant in the neutrinodriven supernova paradigm. Various studies have addressed the "explodability" of supernova progenitors, using 1D simulations and semi-analytic models (e.g., O'Connor \& Ott 2011; Ugliano et al. 2012; Pejcha \& Thompson 2015; Ertl et al. 2016a; Müller et al. 2016; Ebinger et al. 2019; Ertl et al. 2020). Despite variations in detail, the findings suggest that explodability can be gauged by a few key stellar structure parameters. Physically, the key determinants that favor a successful explosion are a low Fe-Si core mass and a low density in the oxygen $(\mathrm{O})$ shell (Ertl et al. 2016a), although other metrics for explodability like the more familiar compactness parameter (O'Connor \& Ott 2011) or the binding energy of the shells outside the Fe-Si core are strongly correlated with these parameters. These structural properties of the core region depend non-monotonically on zero-age main sequence (ZAMS) mass (for single stars), He core mass, and carbon-oxygen $(\mathrm{CO})$ core mass $M_{\mathrm{CO}}$, leaving "islands of explodability" among rather massive progenitors. Nonetheless, from a coarse-grain point of view, there is a trend of decreasing explodability with increasing $\mathrm{He}$ and $\mathrm{CO}$ core mass.

First-principle 3D simulations are not yet capable of surveying stellar explodability in a similar manner as parametrised supernova explosion models. There are tentative indications, however, that neutrino-heating can drive shock expansion in progenitors with cores of high mass and compactness (Chan et al. 2018; Ott et al. 2018; Burrows et al. 2020; Powell \& Müller 2020), which is seemingly in contrast to the previously mentioned findings. These results are not likely to fundamentally overturn the trend toward lower explodability with higher He or CO core mass, since the incipient explosions in progenitors with high-mass cores may be stifled on longer time scales as the shock propagates through the tightly bound massive envelope. If shock revival works effectively in progenitors with high-mass cores, the net effect may merely be to introduce a transition regime of successful explosions with considerable fallback and black-hole formation between the regime of neutron star formation (for less massive cores) and quiet black hole formation (for the most massive cores). This would be compatible with constraints on the progenitors of observed transients from preexplosion images, according to which explosions from highmass progenitors are rare (Smartt 2009, 2015), as well as with the apparent disappearance of massive stars without an explosion (Adams et al. 2017).

Some systematic dependencies of the compact remnant properties can already be discerned from paramaterised and first-principle supernova models. In the case of successful explosions with neutron star formation, there is a rather robust correlation between the progenitor's Fe-Si core mass and the neutron star mass $M_{\mathrm{NS}}$ (e.g., Ertl et al. 2016a; Sukhbold et al. 2016; Müller et al. 2016), because neutrino-driven explosions are often triggered by the drop in mass accretion rate when the collapsing $\mathrm{O}$ shell reaches the stalled shock. The dependence of $M_{\mathrm{NS}}$ on the $\mathrm{He}$ and $\mathrm{CO}$ core mass is not monotonic, but there is a loose positive correlation with significant scatter due to the intricacies of the late stellar evolution stages and of the neutrino-driven mechanism. A linear dependence of $M_{\mathrm{NS}}$ on $M_{\mathrm{CO}}$ with broad scatter would thus seem the most natural first-order approximation.

In case the shock is successfully revived, the explosion energy correlates loosely with core mass and compactness, and with the neutron star mass (Nakamura et al. 2015; Müller et al. 2016, 2019) because the neutron star mass (via the neutron star surface temperature) and the accretion rate determine neutrino emission and neutrino heating. There is also a loose correlation between neutron star (NS) mass, explosion energy, and NS kick velocity since the explosion energy sets the scale for the momentum asymmetry that can be achieved in the innermost ejecta, even though the degree of asymmetry is expected to exhibit stochastic variations (Janka 2017; Vigna-Gómez et al. 2018). 3D simulations indeed indicate substantial scatter in the kicks (Müller et al. 2019; Powell \& Müller 2020), especially for progenitors with low-mass cores (Gessner \& Janka 2018; Stockinger et al. 2020), see e.g., Figure 12 in Müller et al. (2019).

Due to large-scale lepton fraction asymmetries in the proto-neutron star convection zone, anisotropic neutrino emission can also impart kicks of a few tens of $\mathrm{km} \mathrm{s}^{-1}$ onto the neutron star even without pronounced asymmetries in the ejecta (Stockinger et al. 2020).

In the case of black hole formation, the systematics of the remnant properties are less securely understood, partly because there are different scenarios for black hole formation. When a black hole is formed because the shock is never revived, one does not expect any kick, but if a hydrogen envelope is present it may (partly) become unbound as the emission of neutrinos during the proto-neutron star phase decreases the gravitational mass of the star (Nadezhin 1980; Lovegrove \& Woosley 2013; Coughlin et al. 2018). In this case, one can identify black hole mass with the helium core mass as a first-order estimate.

In other cases, a black hole may be formed by fallback after successful shock revival. Fallback can occur via the deceleration of the inner ejecta by the reverse shock (Chevalier 1989), but this mechanism usually only adds $\lesssim 10^{-2} M_{\odot}$ onto the remnant (Ertl et al. 2016b). Substantial fallback can occur, however, if ongoing accretion after shock revival pushes the neutron star to collapse, in which case the dynamics of the fallback is inherently multi-dimensional (Chan et al. 2018, 2020). Based on the work of Chan et al. (2020), one can distinguish two limiting scenarios in this regime of strong fallback.

If the explosion energy is sufficiently high, then the postshock velocity will eventually reach escape velocity even 
though the explosion energy is continuously decreased as the shock sweeps up bound matter. Up to this point, a substantial fraction of the shocked matter will be channelled around the expanding neutrino-heated bubbles and accreted onto the black hole; afterwards shocked material will become unbound and be ejected. Since the expanding bubbles that eventually escape usually develop a unipolar or bipolar structure early in the explosion, they will carry a significant net momentum at the point when accretion freezes out, and the black hole, must by momentum conservation receive a substantial kick that can reach several $100 \mathrm{~km} \mathrm{~s}^{-1}$.

If, on the other hand, the Mach number of the shock comes close to unity as the accumulation of bound matter drains the explosion energy, the blast wave will transition to the weak shock regime, i.e., the shock will propagate as a sonic pulse whose energy is approximately conserved (Mihalas \& Mihalas 1984). As the shock approaches the stellar surface, it will eventually leave the weak shock regime again and shed a part of the envelope. The final mass cut can be estimated by considering when the post-shock velocity eventually matches the escape velocity. The sonic pulse is also spherical in this case, and hence the ejecta momentum and the black hole kick will be small, though residual kicks of order $\mathcal{O}\left(10 \mathrm{~km} \mathrm{~s}^{-1}\right)$ are still possible (Chan et al. 2018).

The transition from the regime of weak fallback to strong and ultimately complete fallback is governed by the ratio of the envelope binding energy to the initial explosion energy. Since the explosion energy is limited to $\sim 2 \times 10^{51} \mathrm{erg}$ in the neutrino-driven paradigm, one would expect that the envelope binding energy (which is correlated with the mass of the oxygen, neon and carbon shells) is the decisive parameter that dictates the amount of fallback.

In reality, fallback is a more complicated, genuinely multi-dimensional hydrodynamic process. Introducing two discrete scenarios with sharp "switches" for terminating fallback allows us to obtain qualitatively correct fallback masses and capture the dependence of fallback on stellar parameters, but likely introduces an overly "clumpy" distribution of remnant masses (as shown in Figure 1) relative to what may be expected in nature. There are as yet too few multidimensional models available to sample the distribution of black hole masses in fallback supernovae, but the more gradual termination of accretion seen in extant multi-D models (Chan et al. 2018, 2020) will likely result in a smoother distribution, and probably a bigger stochastic scatter than predicted by our semi-analytic model. Even though they cannot capture the nuances of multi-dimensional fallback processes, hydrodynamic simulations in spherical symmetry (Ertl et al. 2020) already suggest a slightly broader distribution of black hole masses that reaches down to the most massive neutron star masses and does not exhibit any mass gap.

\subsection{Updated semi-analytic supernova model}

As a basis for more a more agnostic parametrisation of the relation between stellar progenitor parameters and the compact remnant mass and kick, which will be discussed in Section 3, we use results from the semi-analytic supernova model of Müller et al. (2016). This model reproduces much of the phenomenology revealed by the more detailed multi-dimensional simulations. Meanwhile, its parameters have been chosen to comply with observational con- straints (e.g., on supernova explosion energies) that multidimensional simulations do not yet comply with. In particular, the model shows the same loose correlation between progenitor compactness, explosion energy, and the neutron star mass and kick that has been alluded to in our discussion of multi-dimensional simulation in Section 2.1 (Nakamura et al. 2015; Müller et al. 2019; Burrows et al. 2020).

While this model has already been used to formulate fits for neutron star masses and kicks in Vigna-Gómez et al. (2018), the original model of Müller et al. (2016) only included a crude all-or-nothing treatment of fallback, and therefore does not provide useful predictions for black hole masses and kicks. We have therefore updated the semianalytic model to reflect some of the phenomenology of multi-dimensional simulations of black hole formation in fallback supernovae.

During the pre-explosion phase, the updated semianalytic model follows the original prescriptions of Müller et al. (2016) to estimate whether and when shock revival occurs based on scaling laws for the neutrino emission, the quasi-stationary structure of the supernova core, and the neutrino heating conditions. However, we use a slightly smaller value $\zeta=0.7$ for the accretion efficiency for better agreement with the observational constraints on supernova progenitor masses (Smartt 2015). The treatment of the explosion phase has been modified slightly. After shock revival at the initial mass cut $M_{\mathrm{i}}$, the original model computed the explosion energy as a function of mass coordinate by taking into account neutrino heating, nuclear energy release, and the accumulation of bound material by the shock. The final mass cut $M_{\mathrm{f}}$ was determined by the condition that the shock velocity $v_{\text {sh }}$ (estimated from the explosion energy and ejecta mass following Matzner \& McKee 1999) exceeded the escape velocity $v_{\text {esc }}$. If this condition was not met before the gravitational neutron star mass $M_{\text {grav }}$ reached $M_{\max }=2.05 M_{\odot}$, or if the explosion energy dropped to zero, the model assumed that the entire star collapses to a black hole. We have modified this procedure as follows:

(i) Once $M_{\text {grav }}=M_{\max }$, we switch off further energy input by neutrinos, but allow the shock to propagate further after black hole formation, and assume that the explosion energy changes according to the release of nuclear energy by explosive burning and the accumulation of bound matter following Equation (49) of Müller et al. (2016).

(ii) If $v_{\mathrm{sh}}=v_{\mathrm{esc}}$ is reached after black hole formation, we assume that accretion stops. The corresponding mass coordinate defines the final mass cut mass $M_{\mathrm{f}}$. The mass $M_{\text {asym }}=M_{\mathrm{f}}-M_{\mathrm{i}}$ of the strongly asymmetric inner ejecta determines the kick. The explosion energy is still evolved further until shock breakout, or until one of the following conditions is met. This channel corresponds to the scenario of black hole formation with weak fallback in Chan et al. (2020).

(iii) If $v_{\text {sh }}$ drops below the pre-shock sound speed $c_{\mathrm{s}}$ (which is smaller than the escape velocity), we assume a transition to the weak shock regime. In this case, the (small) explosion energy at the transition point is conserved as the shock travels to the surface as a sound pulse, and the final mass cut $M_{\mathrm{f}}$ is determined as the mass coordinate where $v_{\text {sh }}>v_{\text {esc }}$ somewhere further outside. This channel corresponds to the strong fallback scenario in Chan et al. (2020). 
In our supernova model we do not calculate any kick for this channel for want of a suitable analytic theory. The results of Chan et al. $(2018,2020)$ suggest, however, that black hole kicks of several $\times 10 \mathrm{~km} \mathrm{~s}^{-1}$ will be reached.

(iv) Following Lovegrove \& Woosley (2013), we assume that the hydrogen shell (if present) is always ejected, and cap the black hole mass at the helium core mass $M_{\mathrm{He}}$.

Both for neutron stars and black holes, we estimate the typical value of the kick from $M_{\mathrm{f}}$ and $M_{\mathrm{i}}$ as in Vigna-Gómez et al. (2018),

$v_{\text {kick }}=0.16 \frac{\sqrt{E_{\text {expl }}\left(M_{\mathrm{f}}-M_{\mathrm{i}}\right)}}{M_{\text {grav }}}$,

where $E_{\text {expl }}$ is the explosion energy. Since the mass $M_{\mathrm{f}}-M_{\mathrm{i}}$ of the asymmetric inner ejecta is tightly correlated with the explosion energy $E_{\text {expl }}$, this is effectively tantamount to $v_{\text {kick }} \propto E_{\text {expl }}$ in the case of neutron-star forming progenitors with little fallback, though Equation (1) makes the connection to momentum conservation more transparent. Such a scaling of the kick velocity with explosion energy (with some scatter) has indeed been found in self-consistent 3D simulations, see, e.g., Figure 12 in Müller et al. 2019.

To obtain the remnant gravitational mass $M_{\text {grav }}$, we subtract the neutron star binding energy from $M_{\mathrm{f}}$, but no such deduction is made in the case of black hole formation. The stochastic distribution of the kick velocity is not specified any further in the semi-analytic model, and will be dealt with by a parametrised prescription in Section 3. The kick estimate from Equation (1) is best understood as the mode of a broad, probably top-heavy distribution, whose precise shape cannot be predicted based on first principles and a small corpus of $3 \mathrm{D}$ simulations yet.

In practice, $E_{\text {expl }}$ and the mass $M_{\mathrm{f}}-M_{\mathrm{i}}$ of the asymmetric inner ejecta are tightly correlated. Because of the correlation of $E_{\text {expl }}$ with $M_{\mathrm{CO}}$, this suggests a linear dependence of the kick velocity on $M_{\mathrm{CO}}$ as a first-order approximation. For low-mass iron core supernovae and electron capture supernovae, where $M_{\mathrm{NS}} \approx M_{\mathrm{CO}}$, 3D simulations show very small kicks (Müller et al. 2018; Stockinger et al. 2020), and hence it is natural to anchor this approximate linear dependence at zero, i.e., $v_{\text {kick }} \propto \sqrt{E_{\text {expl }}\left(M_{\mathrm{f}}-M_{\mathrm{i}}\right)} \propto\left(M_{\mathrm{CO}}-M_{\mathrm{NS}}\right)$.

We stress that this procedure for estimating fallback after black hole formation rests on a crude simplification of the complex dynamics in multi-dimensional simulations. It does nonetheless qualitatively reproduce the salient features of these simulations; low-mass black holes made in relatively powerful explosion will get sizeable kicks, whereas high-mass black holes formed in weak explosions (which tend to have strong fallback) will not. Unlike the simpler prescriptions for fallback and black hole kicks (Fryer et al. 2012; Spera et al. 2015; Bray \& Eldridge 2016, 2018; Kruckow et al. 2018; Giacobbo \& Mapelli 2020), it is the detailed structure of the innermost shells of the progenitor that determines whether an explosion enters the regime of weak or strong fallback. As a result, our semi-analytic explosion model predicts that in the channel for black hole $(\mathrm{BH})$ formation, weak and strong fallback coexist over a wide range of $\mathrm{CO}$ core masses, and even coexist with the neutron star formation channel. This behaviour is genuinely different from single-valued prescriptions for the remnant properties and significantly changes the mapping from $M_{\mathrm{CO}}$ to the remnant mass (and hence also the remnant kick). The distinct remnant mass "branches" in Figure 1 should be compared, e.g., to Figure 7 in Spera et al. (2015) or Figure 5 in Fryer et al. (2012). This more complex dependence of the remnant properties on $M_{\mathrm{CO}}$ emerges naturally from a more detailed treatment of the supernova explosion physics, not only in our semi-analytic model but also in other studies (e.g., Ugliano et al. 2012; Pejcha \& Thompson 2015; Sukhbold et al. 2016; Ertl et al. 2020). It stands to reason that this complexity will be reflected in the range and distribution of compact remnants and compact binary systems that can be realised in nature, and therefore ought to be taken into account in population synthesis codes.

\section{PARAMETRISED PRESCRIPTION}

Our next goal is to provide a simple prescription with a small number of parameters for the remnant mass and kick as a function of the $\mathrm{CO}$ core and He shell mass of the progenitor (we assume that any hydrogen envelope will always be unbound during the supernova (Lovegrove \& Woosley 2013; Coughlin et al. 2018), if not previously stripped by winds or binary interaction). While we suggest likely values for these parameters based on our semi-analytic model, the exact values should be updated by comparisons with observations.

We assume that the combined process of stellar evolution and supernova explosion and collapse introduces genuine stochasticity on top of deterministic dependencies on stellar parameters. Therefore, unlike all previous recipes, our model is probabilistic in its predictions.

The key parameters governing the mass and kick, along with their default values, are identified in Table 1 . The remnant mass prescription depends predominantly on the $\mathrm{CO}$ core mass, $M_{\mathrm{CO}}$. We split the entire mass range into 5 subranges with boundaries $M_{1}, M_{2}, M_{3}, M_{4}$ depending on the value of $M_{\mathrm{CO}}$.

First, we determine the remnant type. If $M_{\mathrm{CO}}<M_{1}$, the remnant is always a NS. If $M_{1} \leq M_{\mathrm{CO}}<M_{3}$, the remnant is BH with probability $p_{\mathrm{BH}}=\left(M_{\mathrm{CO}}-M_{1}\right) /\left(M_{3}-M_{1}\right)$ and a NS with probability $p_{\mathrm{NS}}=1-p_{\mathrm{BH}}$. If $M_{\mathrm{CO}} \geq M_{3}$, the remnant is always a $\mathrm{BH}$.

If the remnant is a $\mathrm{BH}$, it has a probability $p_{\mathrm{cf}}$ of being formed by complete fallback, in which case the remnant mass is equal to the total He core mass (CO core mass combined with He shell mass). The complete fallback probability is $p_{\mathrm{cf}}=1$ if $M_{\mathrm{CO}} \geq M_{4}$. Otherwise, for $M_{1} \leq M_{\mathrm{CO}} \leq M_{4}$, if the remnant is a black hole, its complete fallback probability is $p_{\mathrm{cf}}=\left(M_{\mathrm{CO}}-M_{1}\right) /\left(M_{4}-M_{1}\right)$. The increasing probability of complete fallback with increasing $M_{\mathrm{CO}}$ is compatible with a higher prevalence of this scenario at high $M_{\mathrm{CO}} \gtrsim 7 M_{\odot}$ in the semi-analytic model (Figure 1).

If the remnant is a $\mathrm{BH}$ but is not formed by complete fallback, the remnant mass follows a normal distribution with mean $\mu_{\mathrm{BH}} M_{\mathrm{CO}}$ and standard deviation $\sigma_{\mathrm{BH}}$.

Inspired by the parametrised semi-analytic model, we introduce several branches for the NS mass. If the remnant is a NS, its mass is given by a normal distribution $N\left(\mu, \sigma^{2}\right)$ where the mean $\mu$ and standard deviation $\sigma$ are:

- If $M_{\mathrm{CO}}<M_{1}$, then $\mu=\mu_{1}$ and $\sigma=\sigma_{1}$.

- If $M_{1} \leq M_{\mathrm{CO}}<M_{2}$, then $\mu=\mu_{2 a}+\mu_{2 b}\left(M_{\mathrm{CO}}-\right.$ $\left.M_{1}\right) /\left(M_{2}-M_{1}\right)$ and $\sigma=\sigma_{2}$. 


\begin{tabular}{|l|l|l|}
\hline Parameter & Default value & Meaning \\
\hline \multicolumn{3}{|c|}{ Mass ranges } \\
$\boldsymbol{M}_{\mathbf{1}}$ & $2.0 M_{\odot}$ & Max CO core mass leading to 100\% NS \\
$\boldsymbol{M}_{\mathbf{2}}$ & $3.0 M_{\odot}$ & Break in NS mass distribution fits \\
$\boldsymbol{M}_{\mathbf{3}}$ & $7.0 M_{\odot}$ & Min CO core mass leading to $100 \% \mathrm{BH}$ \\
$\boldsymbol{M}_{\mathbf{4}}$ & $8.0 M_{\odot}$ & Min CO core mass leading to $100 \%$ fallback \\
\hline \multicolumn{3}{|c|}{ Remnant mass parameters } \\
$\mu_{1}$ & $1.2 M_{\odot}$ & mean NS mass for $M_{\mathrm{CO}}<M_{1}$ \\
$\sigma_{1}$ & $0.02 M_{\odot}$ & NS mass scatter for $M_{\mathrm{CO}}<M_{1}$ \\
$\mu_{2 a}$ & $1.4 M_{\odot}$ & NS mass offset for $M_{1} \leq M_{\mathrm{CO}}<M_{2}$ \\
$\mu_{2 b}$ & 0.5 & NS mass scaling for $M_{1} \leq M_{\mathrm{CO}}<M_{2}$ \\
$\sigma_{2}$ & $0.05 M_{\odot}$ & NS mass scatter for $M_{1} \leq M_{\mathrm{CO}}<M_{2}$ \\
$\mu_{3 a}$ & $1.4 M_{\odot}$ & NS mass offset for $M_{2} \leq M_{\mathrm{CO}}<M_{3}$ \\
$\mu_{3 b}$ & 0.4 & NS mass scaling for $M_{2} \leq M_{\mathrm{CO}}<M_{3}$ \\
$\sigma_{3}$ & $0.05 M_{\odot}$ & NS mass scatter for $M_{2} \leq M_{\mathrm{CO}}<M_{3}$ \\
$\mu_{\mathrm{BH}}$ & 0.8 & BH mass scaling for $M_{1} \leq M_{\mathrm{CO}}<M_{4}$ \\
$\sigma_{\mathrm{BH}}$ & $0.5 M_{\odot}$ & BH mass scatter for $M_{1} \leq M_{\mathrm{CO}}<M_{4}$ \\
$M_{\mathrm{NS}, \min }$ & $1.13 M_{\odot}$ & minimal NS mass from core-collapse SN \\
$\boldsymbol{M}_{\mathrm{NS}, \max }$ & $2.0 M_{\odot}$ & maximal NS mass \\
\hline \multicolumn{3}{|l|}{ Natal kick parameters } \\
$\boldsymbol{v}_{\mathrm{NS}}$ & $400 \mathrm{~km} \mathrm{~s}{ }^{-1}$ & NS kick scaling prefactor \\
$\boldsymbol{v}_{\mathrm{BH}}$ & $200 \mathrm{~km} \mathrm{~s} \mathrm{~s}^{-1}$ & BH kick scaling prefactor \\
$\sigma_{\mathrm{kick}}$ & 0.3 & fraction kick scatter \\
\hline
\end{tabular}

Table 1. The list of parameters describing our remnant mass and kick model. The most uncertain parameters that can benefit from observational constraints appear in bold.

- If $M_{2} \leq M_{\mathrm{CO}}<M_{3}$, then $\mu=\mu_{3 a}+\mu_{3 b}\left(M_{\mathrm{CO}}-\right.$ $\left.M_{2}\right) /\left(M_{3}-M_{2}\right)$ and $\sigma=\sigma_{3}$.

We assume that NSs have a minimum mass of $M_{\mathrm{NS}, \mathrm{min}}$ and a maximum mass of $M_{\mathrm{NS} \text {,max }}$, and therefore re-draw any remnant mass guesses that fall outside this allowed range (and similarly for any $\mathrm{BH}$ mass guesses below $M_{\mathrm{NS} \text {,max }}$ ).

The remnant mass prescription above is already a recipe for the gravitational mass of neutron stars. For black holes, we expect that only $0.1 M_{\odot} c^{2} \sim$ few $\times 10^{53}$ erg of energy can be lost in neutrinos during the collapse. This follows from the assumption that the total post-bounce neutrino luminosity is of order $10^{53} \mathrm{erg} \mathrm{s}^{-1}$ and the BH formation occurs on a timescale of a few seconds (Mirizzi et al. 2016; Chan et al. 2018), which is substantially shorter than the KelvinHelmholtz timescale of the proto-neutron star. Therefore, all final BH remnant masses should be reduced by at most $0.1 M_{\odot}$ from the estimates given above, a correction which we ignore given the other uncertainties present (cf. the much larger $10 \%$ mass loss assumed by Fryer et al. (2012)).

Although all simulated stars were single stars, we assume that binary interactions do not significantly impact the core structure, and therefore all prescriptions can be carried over directly to stars in interacting binaries, including those whose helium envelopes had been stripped by previous interactions (e.g., case BB mass transfer leading to ultra-stripped supernovae, Tauris et al. 2015).

We follow the prescription of Hurley et al. (2000) with partial amendments from Belczynski et al. (2008) and Fryer et al. (2012) to determine which stars undergo electroncapture and regular core-collapse supernovae. Stars with core masses above $2.25 M_{\odot}$ at the base of the asymptotic giant branch undergo core-collapse supernovae once their carbon-oxygen core reaches the mass threshold of Eq. (75) of Hurley et al. (2000), where we use $1.38 M_{\odot}$ in lieu of the Chandrasekhar mass. The remnant masses are then computed as described above. If the helium core mass is below
$2.25 M_{\odot}$ but above $1.6 M_{\odot}$ at the base of the asymptotic giant branch and $M_{\mathrm{CO}}$ reaches $1.38 M_{\odot}$ during subsequent evolution, the star is assumed to form a NS with a gravitational mass of $1.26 M_{\odot}$ in an electron-capture supernova.

Our models suggest that the inner ejecta during supernovae leading to NS formation, which are coupled to the remnant and can therefore provide an asymmetric natal kick, have a similar degree of asymmetry and a similar velocity across all simulations. Outer ejecta, including most of the helium envelope, are expelled before significant asymmetry is built up and hence do not provide a natal kick, while any material that falls back cannot provide a kick. Conservation of momentum implies that $M_{\mathrm{NS}} v_{\text {kick }}=\alpha\left(M_{\mathrm{CO}}-M_{\mathrm{NS}}\right) v_{\mathrm{ej}}$, where $\alpha$ is the asymmetry parameter, $v_{\mathrm{ej}}$ is the typical ejecta velocity, and $M_{\mathrm{CO}}-M_{\mathrm{NS}}$ is a proxy for the mass of the material ejected asymmetrically while there is significant coupling with the remnant (see Section 2.2). Therefore, our model for the mean natal kick for neutron stars is

$\mu_{\text {kick }}=v_{\mathrm{NS}} \frac{M_{\mathrm{CO}}-M_{\mathrm{NS}}}{M_{\mathrm{NS}}}$.

We note that the physical reason for this scaling lies in the dependence of the explosion energy on CO core mass, but for the purpose of constructing a parametrised recipe, it is immaterial whether there is a direct or and indirect dependence on $M_{\mathrm{CO}}$. We allow for stochasticity by adding an additional component drawn from a Gaussian distribution with standard deviation $\sigma_{\text {kick }} \mu_{\text {kick }}$.

Bray \& Eldridge (2016, 2018); Giacobbo \& Mapelli (2020) previously proposed the use of momentum-preserving natal kick recipes in population synthesis studies. These recipes have a generally similar form to that of Eq. (2), but with one significant distinction: they use the ratio of the ejecta mass to the remnant mass to determine the kick, while we use the ratio of the ejected carbon-oxygen core mass to the remnant. Our models indicate that this is a better proxy for the portion of the ejecta that are coupled to the asymme- 
try which builds up during the later stages of the collapse. Estimating the kick based on the total ejecta mass would suggest substantially larger kicks for non-stripped supernova progenitors, which is sharply contradicted by 3D simulations Müller et al. (2019). Moreover, Bray \& Eldridge (2016, 2018) fits include an offset to the scaling with this ratio, and in the latter case allow for negative kick velocities, i.e., remnant velocities in the direction in which greater momentum is carried away. While Bray \& Eldridge (2018) explain this as being sourced by the gravitational tugboat mechanism (Janka 2017), this appears to unphysically contradict conservation of momentum. Finally, we consider stochasticity in the kicks to be an intrinsic feature of stellar evolution and supernova instabilities, and our kick models reflect this along with the remnant mass models.

We do not use a distinct prescription to provide reduced natal kicks for electron-capture or ultra-stripped supernovae, as the low $\mathrm{CO}$ core masses of their progenitors already provide the necessary kick reduction (Tauris et al. 2015; Beniamini \& Piran 2016; Vigna-Gómez et al. 2018).

Observations of black-hole X-ray binaries and microlensed black holes indicate that relatively low-mass black holes may experience kicks of a few tens to $\sim 100 \mathrm{~km}$ $\mathrm{s}^{-1}$ (Willems et al. 2005; Fragos et al. 2009; Mandel 2016; Mirabel 2017; Wyrzykowski \& Mandel 2020; Atri et al. 2019) and possibly even a few hundred $\mathrm{km} \mathrm{s}^{-1}$ (Repetto et al. 2017). Motivated by the discussion in Section 2, we use the same functional form for black hole kicks as for neutron star kicks (Eq. (2)), but with a reduced prefactor $v_{\mathrm{BH}}$ instead of $v_{\mathrm{NS}}$ :

$\mu_{\text {kick }}=v_{\mathrm{BH}} \frac{\max \left(M_{\mathrm{CO}}-M_{\mathrm{BH}}, 0\right)}{M_{\mathrm{BH}}}$.

When a black hole is formed through complete fallback, $M_{\mathrm{CO}}-M_{\mathrm{BH}} \leq 0$, so such black holes do not experience a natal kick in our model.

Remnant kick magnitudes are constrained to be positive and are re-drawn if the initial guess is negative.

\section{SINGLE-STELLAR EVOLUTION}

In Figure 1, we compare the remnant masses as a function of progenitor $\mathrm{CO}$ core masses as predicted by a combination of the detailed stellar evolution and the semi-analytic supernova model described in Section 2 against the probabilistic parametrised recipes introduced in Section 3. We implemented the stochastic recipes in the COMPAS rapid population synthesis code (Stevenson et al. 2017; Vigna-Gómez et al. 2018).

The recipes generally reproduce the key features of the more detailedsemi-analytic supernova model: the probabilistic nature of the remnant as a NS or $\mathrm{BH}$ and the approximate branching ratio of the two outcomes as a function of $\mathrm{CO}$ core mass; the approximate fraction of black holes undergoing complete fallback; and the two strands of the NS mass distribution.

Some of the fine features of the detailed models are, of course, not reproduced by the simplified recipes. In certain cases (e.g., the difference in remnant mass for the most massive black holes that undergo complete fallback), this is due to differences between the pre-supernova models of Kepler (Weaver et al. 1978) used for the detailed simulations

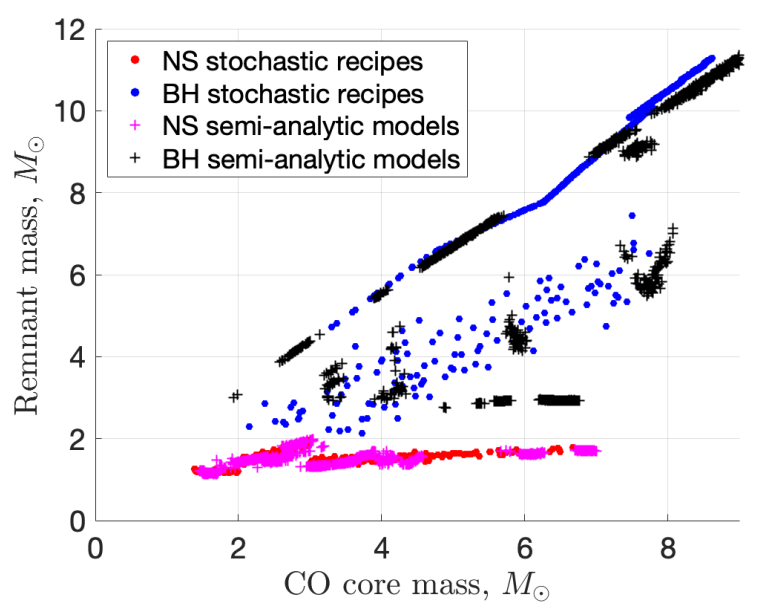

Figure 1. Remnant masses as a function of CO core masses from single-star simulations: a comparison between detailed stellar evolution coupled with the semi-analytic supernova model against COMPAS stellar evolution and our stochastic recipes.

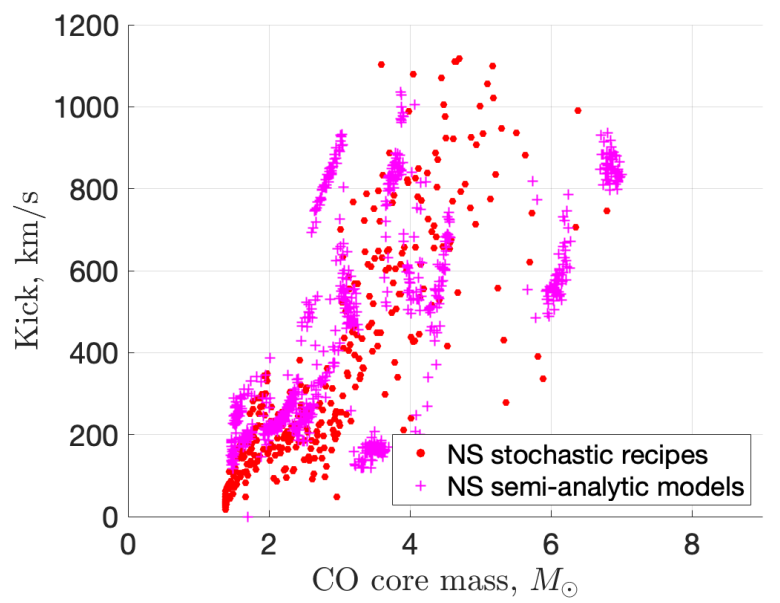

Figure 2. Neutron star natal kicks (3D velocities) as a function of $\mathrm{CO}$ core masses from single star simulations: a comparison between detailed stellar evolution coupled to the semianalytic supernova model against COMPAS stellar evolution and our stochastic recipes.

vs. the single-stellar evolution models of Hurley et al. (2000) employed in COMPAS. Other fine features of detailed models are generally sufficiently uncertain at the moment that attempting to reproduce them appears pre-mature.

For example, the detailed models show an island of consistent $\mathrm{BH}$ formation between $\mathrm{CO}$ core masses of $4.6 M_{\odot}$ and $6 M_{\odot}$, whereas the stochastic fit assumes a gradual increase of the $\mathrm{BH}$ formation probability over a wider range in $M_{\mathrm{CO}}$. This island can be traced to a peak in the core compactness of the progenitor models that is associated with the transition from convective to radiative carbon burning (Sukhbold et al. 2018). While this peak appears quite robust in singlestar models, its location and width are, however, sensitive to details like the neutrino cooling rates (Müller et al. 2016), 
the carbon-to-oxygen ratio after helium burning (Schneider et al. 2020) (which may be affected by previous mass transfer episodes), and conceivably to other uncertainties in stellar models.

Another example concerns the presence or absence of a gap between NS and BH masses. While the detailed simulations support a narrow mass gap between the most massive neutron stars $\left(\approx 1.99 M_{\odot}\right)$ and the lightest black holes $\left(\approx 2.75 M_{\odot}\right)$, this gap is not enforced in the stochastic recipes. This is motivated by the known limitations of our semi-analytic treatment of fallback that likely results in an overly clumpy distribution of black hole masses as discussed in Section 2. Hydrodynamic models of fallback suggest a remnant mass distribution without a gap (Ertl et al. 2020). If the theoretical models (especially multi-D simulations) reach greater clarity on the presence or absence of a gap, the recipes can be updated in the future.

We show the neutron star natal kicks as a function of progenitor $\mathrm{CO}$ core masses in figure 2 . The kicks do not reproduce the semi-analytic models very closely, particularly at the lowest $\mathrm{CO}$ core masses, where the recipe scaling of the kick with the ratio of the $\mathrm{CO}$ ejecta mass to the remnant mass, equation (2), under-predicts the kicks relative to detailed simulations and asymptotes to zero for the least massive cores. As argued previously, this is justified by the premise behind our stochastic recipes: The overall trends in the kick velocity predicted by the semi-analytic model are likely robust, whereas the fine-grained structure in the predicted distribution likely depends on more uncertain details in the underlying stellar evolution and explosion models. The different asymptotic behaviour at low core masses is intentional, however, since it is line with the results of more sophisticated multi-D simulations that cannot be easily reproduced in the semi-analytic model. For the lowest $\mathrm{CO}$ core masses, the explosion develops too quickly for significant global asymmetries to grow, i.e. the assumption of a universal asymmetry parameter breaks down, and kicks may hence be as low as a few $\mathrm{km} \mathrm{s}^{-1}$. The lower kick values allowed by the simplified recipes are also motivated by indications of reduced supernova kicks from low iron-core mass progenitors (Podsiadlowski et al. 2004).

We can now investigate the predicted distributions of remnant masses and natal kicks in a population of single stars by integrating over the initial mass function. We assume solar metallicity ( $Z=0.0142$, Asplund et al. 2009) and the standard COMPAS wind prescription for pre-supernova evolution. We evolve stars with zero-age main sequence masses from $6 M_{\odot}$ (lower masses always leave $\mathrm{CO}$ white dwarfs behind) to $36 M_{\odot}$ (higher masses always lead to a complete collapse into black holes) following the Kroupa (2001) initial mass function, $p(M) \propto M^{-2.3}$ in this mass range. The resulting remnant mass distribution is plotted in figure 3, along with the Kroupa initial mass function to guide the eye.

The corresponding kick distribution, separated into NS and BH kicks, is shown in figure 4 . Two thirds of the initialmass-function-weighted black holes for progenitors in this mass range receive zero natal kicks; the natal kicks of kicked BHs (shown in red) range up to $\sim 150 \mathrm{~km} \mathrm{~s}^{-1}$ with a rootmean-square kick of $\sim 60 \mathrm{~km} \mathrm{~s}^{-1}$.

Neutron stars get larger kicks (blue), as expected, following a broad distribution extending from $\sim 15$ to $\sim 1500$

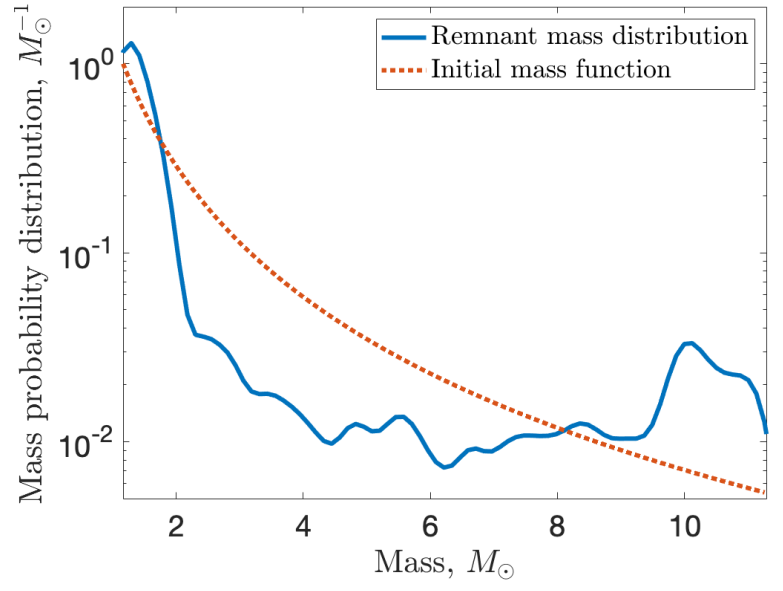

Figure 3. Remnant mass probability distribution (blue) for stars sampled from the initial mass function (dashed red).

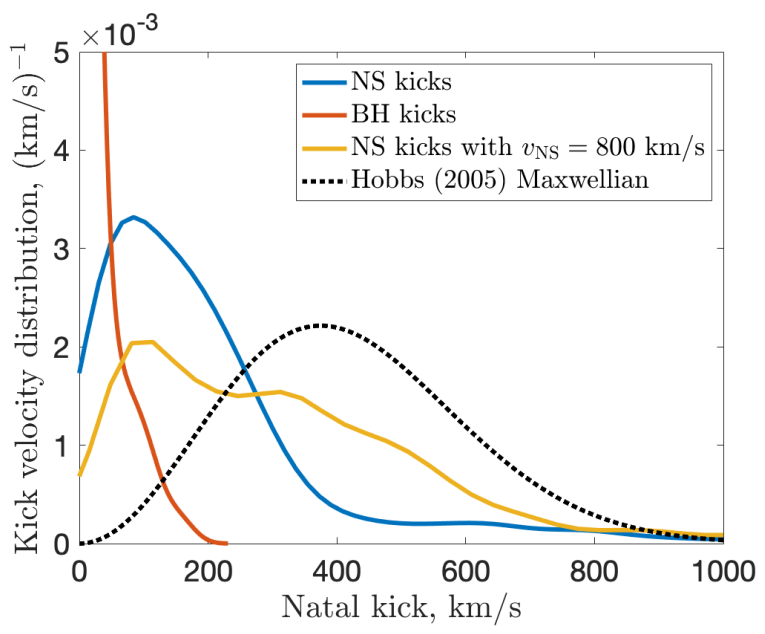

Figure 4. Natal kick probability distribution for neutron stars (blue) and black holes (red) for stars sampled from the initial mass function. Neutron star kick distribution assuming $v_{\mathrm{NS}}=800 \mathrm{~km}$ $\mathrm{s}^{-1}$ is shown in yellow, while a Maxwellian distribution with a central parameter of $265 \mathrm{~km} \mathrm{~s}^{-1}$ (Hobbs et al. 2005) is displayed in dashed black.

$\mathrm{km} \mathrm{s}^{-1}$, with a root-mean-square velocity of $\sim 270 \mathrm{~km} \mathrm{~s}^{-1}$ but a mode of only $\sim 80 \mathrm{~km} \mathrm{~s}^{-1}$. This is lower than the Maxwellian distribution with a central parameter of $265 \mathrm{~km}$ $\mathrm{s}^{-1}$ proposed by Hobbs et al. (2005) (plotted in a black dotted curve in figure 4 for comparison). However, the observed pulsar velocity distribution is uncertain, and a number of other kick distributions have been fitted to radio pulsar observations (e.g., Arzoumanian et al. 2002; Verbunt et al. 2017). In particular, distance estimates based on dispersion measures are unreliable and could be systematically biased (Deller et al. 2019), leading to errors in the inferred pulsar velocities. Furthermore, there may be significant selection effects in the observed sample, ranging from potential correlations between velocity and detectability to only more rapidly kicked neutron stars escaping from binaries where 
many pulsars are born. In any case, the parametrisation of our kick distribution allows for ready adjustment (e.g., the yellow line in figure 4 corresponds to $v_{\mathrm{NS}}=800 \mathrm{~km} \mathrm{~s}^{-1}$ rather than the default guess of $v_{\mathrm{NS}}=400 \mathrm{~km} \mathrm{~s}^{-1}$ ) and we leave observational constraints on the parameter values for a future investigation.

Our model is consistent with the observation of large numbers of neutron stars in globular clusters. These have a typical escape velocity of $\sim 50 \mathrm{~km} \mathrm{~s}^{-1}$, much smaller than most proposed kick velocity distributions, leading to the expectation that most neutron stars would be ejected at birth (e.g., Lyne \& Lorimer 1994). If natal kicks are drawn from the Hobbs et al. (2005) fit, fewer than $0.2 \%$ of all neutron stars would get kicks below $50 \mathrm{~km} \mathrm{~s}^{-1}$ and be retained in globular clusters. Meanwhile, the significant tail of low natal kicks in our model implies that $20 \%$ of all neutron stars (and $30 \%$ of all compact remnants) should be retained. Even increasing $v_{\mathrm{NS}}$ to $800 \mathrm{~km} \mathrm{~s}^{-1}$, about $3 \%$ of all neutron stars formed in globular clusters would remain there. Here, we treat all stars as single and neglect the impact of binary companions on the retention fraction, either through increasing the effective inertial mass of a tight binary or through binary interactions that enhance the fraction of low-kick supernovae.

\section{CONCLUSION}

We presented a set of recipes for compact-object remnant masses and natal kicks. These recipes are derived from an examination of recent findings from parametrised supernova models and first-principle 3D models. They are probabilistic, accounting for the intrinsic stochasticity of stellar evolution and supernovae in the spread of outcomes (neutron stars or black holes) and the mass and kick values. These recipes will be particularly useful for rapid stellar and binary population synthesis. As an illustrative example, we showed the consequences of applying the recipes to the evolution of single massive stars.

Some of the key features of our prescriptions qualitatively match the observations. For example, the absence of a mass gap between neutron stars and black holes ${ }^{1}$ in the recipes is consistent with microlensing observations Wyrzykowski \& Mandel (2020). The apparent mass gap from the analysis of black hole X-ray binaries (Özel et al. 2010; Farr et al. 2011) could be due to the evolutionary history of such systems or observational biases (Kreidberg et al. 2012). The non-negligible tail of low-kick neutron stars may help explain the retention of neutron stars in globular clusters, a challenge for high-kick models given typical escape velocities of $\lesssim 50 \mathrm{~km} \mathrm{~s}^{-1}$.

The recipes contain 19 free parameters, of which 7 (highlighted in bold in table 1) are likely to be particularly uncertain and important. While the supernova models provide guidance on their values, they do not uniquely determine them. These free parameters should be constrained

1 While this paper was under review, Abbott et al. (2020) announced the gravitational-wave discovery of a merging compact object binary, GW190814, whose lighter companion has a mass of $2.6 \pm 0.1 \mathrm{M}_{\odot}$, placing in this putative mass gap. through a consistent application of all available observational evidence, including the masses and kicks of Galactic neutron stars observed as radio pulsars; the masses and velocities of neutron-star and black-hole X-ray binaries, including Be X-ray binaries; the retention of neutron stars and black holes in star clusters and globular clusters; microlensing measurements; and compact-object merger rates and properties determined from gravitational waves, short gamma-ray bursts, kilonovae, and r-process nucleosynthesis observations.

\section{ACKNOWLEDGEMENTS}

We thank Ryosuke Hirai, Jeff Riley, Heloise Stevance, Simon Stevenson, Alejandro Vigna-Gomez, Reinhold Willcox, and other members of COMPAS and BPASS teams for useful discussions and suggestions. IM and BM are recipient of the Australian Research Council Future Fellowships FT190100574 and FT160100035, respectively.

\section{DATA AVAILABILITY}

The data underlying this article will be shared on reasonable request to the authors. Simulations in this paper made use of the COMPAS rapid population synthesis code which is freely available at http://github.com/TeamCOMPAS/COMPAS.

\section{REFERENCES}

Abbott R., Abbott T. D., Abraham S., Acernese F., et al., 2020, ApJ, 896, L44

Adams S. M., Kochanek C. S., Gerke J. R., Stanek K. Z., Dai X., 2017, MNRAS, 468, 4968

Arzoumanian Z., Chernoff D. F., Cordes J. M., 2002, ApJ, 568, 289

Asplund M., Grevesse N., Sauval A. J., Scott P., 2009, ARA\&A, 47, 481

Atri P., et al., 2019, MNRAS, 489, 3116

Belczynski K., Kalogera V., Rasio F. A., Taam R. E., Zezas A., Bulik T., Maccarone T. J., Ivanova N., 2008, ApJSupplement, 174, 223

Belczynski K., Wiktorowicz G., Fryer C. L., Holz D. E., Kalogera V., 2012, ApJ, 757, 91

Beniamini P., Piran T., 2016, MNRAS, 456, 4089

Bray J. C., Eldridge J. J., 2016, MNRAS, 461, 3747

Bray J. C., Eldridge J. J., 2018, MNRAS, 480, 5657

Burrows A., 2013, Reviews of Modern Physics, 85, 245

Burrows A., Radice D., Vartanyan D., Nagakura H., Skinner M. A., Dolence J. C., 2020, MNRAS, 491, 2715

Chan C., Müller B., Heger A., Pakmor R., Springel V., 2018, ApJ, 852, L19

Chan C., Mueller B., Heger A., 2020, arXiv e-prints, p. arXiv:2003.04320

Chevalier R. A., 1989, ApJ, 346, 847

Clausen D., Piro A. L., Ott C. D., 2015, ApJ, 799, 190

Cordes J. M., Chernoff D. F., 1998, ApJ, 505, 315

Coughlin E. R., Quataert E., Fernández R., Kasen D., 2018, MNRAS, 477, 1225

Deller A. T., et al., 2019, ApJ, 875, 100

Ebinger K., Curtis S., Fröhlich C., Hempel M., Perego A., Liebendörfer M., Thielemann F.-K., 2019, ApJ, 870, 1

Ertl T., Janka H. T., Woosley S. E., Sukhbold T., Ugliano M., 2016a, ApJ, 818, 124 
Ertl T., Ugliano M., Janka H.-T., Marek A., Arcones A., 2016b, ApJ, 821, 69

Ertl T., Woosley S. E., Sukhbold T., Janka H. T., 2020, ApJ, 890, 51

Farr W. M., Kremer K., Lyutikov M., Kalogera V., 2011, ApJ, 742,81

Faucher-Giguère C.-A., Kaspi V. M., 2006, ApJ, 643, 332

Fragos T., Willems B., Kalogera V., Ivanova N., Rockefeller G., Fryer C. L., Young P. A., 2009, ApJ, 697, 1057

Fryer C. L., Belczynski K., Wiktorowicz G., Dominik M., Kalogera V., Holz D. E., 2012, ApJ, 749, 91

Gessner A., Janka H.-T., 2018, ApJ, 865, 61

Giacobbo N., Mapelli M., 2020, ApJ, 891, 141

Hansen B. M. S., Phinney E. S., 1997, MNRAS, 291, 569

Hobbs G., Lorimer D. R., Lyne A. G., Kramer M., 2005, MNRAS, 360,974

Hurley J. R., Pols O. R., Tout C. A., 2000, MNRAS, 315, 543

Hwang U., Laming J. M., 2012, ApJ, 746, 130

Janka H.-T., 2012, Annual Review of Nuclear and Particle Science, 62,407

Janka H.-T., 2017, ApJ, 837, 84

Kreidberg L., Bailyn C. D., Farr W. M., Kalogera V., 2012, ApJ, 757,36

Kroupa P., 2001, MNRAS, 322, 231

Kruckow M. U., Tauris T. M., Langer N., Kramer M., Izzard R. G., 2018, MNRAS, 481, 1908

Lovegrove E., Woosley S. E., 2013, ApJ, 769, 109

Lyne A. G., Lorimer D. R., 1994, Nature, 369, 127

Mandel I., 2016, MNRAS, 456, 578

Matzner C. D., McKee C. F., 1999, ApJ, 510, 379

Mihalas D., Mihalas B. W., 1984, Foundations of radiation hydrodynamics. Oxford University Press

Mirabel F., 2017, New Astronomy Reviews, 78, 1

Mirizzi A., Tamborra I., Janka H. T., Saviano N., Scholberg K., Bollig R., Hüdepohl L., Chakraborty S., 2016, Nuovo Cimento Rivista Serie, 39, 1

Müller B., 2020, Living Reviews in Computational Astrophysics, 6,3

Müller B., Heger A., Liptai D., Cameron J. B., 2016, MNRAS, 460,742

Müller B., Gay D. W., Heger A., Tauris T. M., Sim S. A., 2018, MNRAS, 479, 3675

Müller B., et al., 2019, MNRAS, 484, 3307

Nadezhin D. K., 1980, Ap\&SS, 69, 115

Nakamura K., Takiwaki T., Kuroda T., Kotake K., 2015, PASJ, 67, 107

O'Connor E., Ott C. D., 2011, ApJ, 730, 70

Ott C. D., Roberts L. F., da Silva Schneider A., Fedrow J. M., Haas R., Schnetter E., 2018, ApJ, 855, L3

Özel F., Psaltis D., Narayan R., McClintock J. E., 2010, ApJ, 725,1918

Patton R. A., Sukhbold T., 2020, arXiv e-prints, p. arXiv:2005.03055

Pejcha O., Thompson T. A., 2015, ApJ, 801, 90

Podsiadlowski P., Langer N., Poelarends A. J. T., Rappaport S., Heger A., Pfahl E., 2004, ApJ, 612, 1044

Powell J., Müller B., 2020, arXiv e-prints, p. arXiv:2002.10115

Repetto S., Igoshev A. P., Nelemans G., 2017, MNRAS, 467, 298

Sana H., et al., 2012, Science, 337, 444

Schneider F. R. N., Podsiadlowski P., Müller B., 2020, arXiv eprints, p. arXiv:2008.08599

Smartt S. J., 2009, ARA\&A, 47, 63

Smartt S. J., 2015, Publ. Astron. Soc. Australia, 32, e016

Spera M., Mapelli M., Bressan A., 2015, MNRAS, 451, 4086

Stevenson S., Vigna-Gómez A., Mandel I., Barrett J. W., Neijssel C. J., Perkins D., de Mink S. E., 2017, Nature Communications, 8, 14906

Stockinger G., et al., 2020, arXiv e-prints, p. arXiv:2005.02420
Sukhbold T., Ertl T., Woosley S. E., Brown J. M., Janka H.-T., 2016, ApJ, 821, 38

Sukhbold T., Woosley S. E., Heger A., 2018, ApJ, 860, 93

Tauris T. M., Langer N., Podsiadlowski P., 2015, MNRAS, 451, 2123

Ugliano M., Janka H.-T., Marek A., Arcones A., 2012, ApJ, 757, 69

Van Dyk S. D., 2017, Philosophical Transactions of the Royal Society of London Series A, 375, 20160277

Verbunt F., Igoshev A., Cator E., 2017, A\&A, 608, A57

Vigna-Gómez A., et al., 2018, MNRAS, 481, 4009

Weaver T. A., Zimmerman G. B., Woosley S. E., 1978, ApJ, 225, 1021

Willems B., Henninger M., Levin T., Ivanova N., Kalogera V., McGhee K., Timmes F. X., Fryer C. L., 2005, ApJ, 625, 324

Wyrzykowski Ł., Mandel I., 2020, A\&A, 636, A20 